\title{
Carbon budget for a coastal inlet in relation to intensive cultivation of suspension-feeding bivalve molluscs
}

\author{
Paul G. Rodhouse* \& Cilian M. Roden \\ Shellfish Research Laboratory, Carna, Co. Galway, Ireland
}

\begin{abstract}
Killary Harbour, a fjordic inlet on the west coast of Ireland, is the site of intensive suspended cultivation of mussels. Data on biomass and production of the macrobenthic infauna and zooplankton in the system are combined with literature data on primary production and wild and cultured mussel production in Killary Harbour to produce a balanced carbon budget. Total particulate carbon input to the system is 1907 tonnes ${\mathrm{C} \mathrm{yr}^{-1}}^{-}$. Carbon production by phytoplankton is partitioned between zooplankton, cultured mussels, wild mussels, phytoplankton respiration and the detritus pool. The detritus pool also receives inputs from river carbon, macroalgae, zooplankton faeces, mussel faeces and phytoplankton. There is a carbon deficit which is probably accounted for by DOC input in freshwater, or import of marine $\mathrm{POC}$, which were not measured. The data are used to estimate the maximum potential yield of cultured mussels from the system. This is in the order of 2000 to 3000 tonnes live weight per year. At this level of production, changes in the ecosystem are predicted. These are expected to arise from competition between mussels and zooplankton for food resources, increased sedimentation in localized areas, and disruption of the nitrogen cycle. However, nitrogen should not limit mussel production. Mussel culture, unlike fish culture in sea cages, re-routes the flow of energy and materials in coastal marine ecosystems but does not cause eutrophication by nutrient input. This is discussed in relation to red tides.
\end{abstract}

\section{INTRODUCTION}

Killary Harbour is one of the principal sites in Ireland for intensive suspended cultivation of the mussel Mytilus edulis, as well as being the chief area for seeding ropes (Rodhouse et al. 1984a). The mariculture industry is expanding and this raises the question of carrying capacity and potential yield of cultured, suspension-feeding bivalve molluscs in coastal marine sites. The Shellfish Research Laboratory has addressed this question by carrying out an investigation of carbon and nutrient flow in wild and cultured mussel populations, in relation to carbon and nutrient flow in the Killary Harbour system (Rodhouse et al. 1984b, 1985, McMahon \& Patching 1984).

Potential bivalve yield in coastal water, if dependent

\footnotetext{
- Present address: British Antarctic Survey, Natural Environment Research Council, High Cross, Madingley Road, Cambridge CB3 OET, United Kingdom
}

on in situ primary production of phytoplankton, averages about 0.15 tonnes $\mathrm{ha}^{-1} \mathrm{yr}^{-1}$ dry meat weight (= approximately $4 \%$ live weight). However, intensive cultivation in small areas has produced 5 to 500 tonnes ha ${ }^{-1} \mathrm{yr}^{-1}$ (Ryther 1969). These high values result from the concentration into a small area, such as an estuary or fjord, of organic matter produced over a large area. Bivalve production depends upon the food level in the water and the rate at which it is carried to the animals by tidal currents. Raft culture may be more effective in bringing food to the animals, by about 3 orders of magnitude, but since high productivity of bivalves depends upon the level of food produced over a large area, production measured on, for example, a small experimental raft or rafts cannot be extrapolated for the entire area of a mariculture site such as Killary Harbour.

In this paper we present data on the carbon budget for the Killary Harbour system which will be used to estimate the carrying capacity of the inlet for intensive cultivation of mussels. 


\section{MATERIALS AND METHODS}

Killary Harbour is a fjordic inlet on the west coast of Ireland. Leenane, the only village on the inlet, is positioned at $53^{\circ} 57^{\prime} \mathrm{N}, 9^{\circ} 42^{\prime} \mathrm{W}$. The inlet is approximately $11 \mathrm{~km}$ in length with an average breadth of about $700 \mathrm{~m}$ and an average depth of about $14 \mathrm{~m}$. The deepest part is $45 \mathrm{~m}$ immediately inside Inishbarna, an island on the entrance sill. For the purpose of this study the inlet was divided into 5 sectors, $A$ to $E$, which are shown in Fig. 1. The dimensions of each sector are given in Table 1. The total catchment area of Killary Harbour is some $250 \mathrm{~km}^{2} ; 2$ rivers, the Erriff and Bundorragha, drain mountainous bogland.

Primary production and allochthonous carbon sources. Measurement and estimation of phytoplankton production and respiration, riverine discharge of particulate organic carbon (POC) plus 'active' dissolved organic carbon (DOC), and sedimentation and flushing of organic carbon within the Killary Harbour system were described by McMahon \& Patching (1984).

Macroalgae cover a small area in Killary Harbour because of rapidly shelving rocky shores or the presence of unstable sand and gravel. Assuming that a 10 $\mathrm{m}$ wide strip along $23 \mathrm{~km}$ of coastline is covered by

Table 1. Surface area and mean depth of Killary Harbour, Ireland

\begin{tabular}{|ccc|}
\hline Sector & Surface area $\left(\mathrm{m}^{2}\right)$ & Mean depth $(\mathrm{m})$ \\
\hline A & $1.34 \times 10^{6}$ & 4.04 \\
B & $1.41 \times 10^{6}$ & 11.29 \\
C & $2.17 \times 10^{6}$ & 14.81 \\
D & $1.43 \times 10^{6}$ & 18.95 \\
E & $9.55 \times 10^{5}$ & 23.87 \\
Total & $7.29 \times 10^{6}$ & - \\
Mean & - & 14.15 \\
\hline
\end{tabular}

macroalgae (Lambe, cited in Keegan \& Mercer 1986), an area of $23 \times 10^{4} \mathrm{~m}^{2}$ is available for seaweed production. Production rate by macroalgae Laminaria spp. is reported to be between 100 and $400 \mathrm{~g} \mathrm{C} \mathrm{m}^{-2} \mathrm{yr}^{-1}$ (Raymont 1980).

Benthic microalgal production was estimated to be $7 \mathrm{~g} \mathrm{C} \mathrm{m}^{-2} \mathrm{yr}^{-1}$. This is the value given by Raine \& Patching (1980) for Roskeeda Bay, further south on the Irish coast, where similar conditions exist of relatively deep water $(10$ to $20 \mathrm{~m})$ and fine, easily disturbed sediment.

Zooplankton. Sampling methods and the seasonal cycle of abundance of zooplankton in Killary Harbour are given by Ryan et al. (1986).

For copepods, standing crop biomass was estimated for Acartia clausii copepodites and adults from lengthweight equations given by Durbin \& Durbin (1978) and Robertson (1968); for Oithona nana and $O$. similis from the equation given by Evans (1977); for all other copepods - mostly Paracalanus, Pseudocalanus, Centropages, Temora spp. and copepodites - from the equation given by Robertson (1968) and for copepod naupli from the equation given by Durbin \& Durbin (1978). Estimates of dry weight were converted to carbon assuming carbon content to be 0.5 (Omori 1969). Production was estimated using daily production: biomass (P:B) ratios given by Greze et al. (1968), Greze (1978) and Uye (1982). Where more than one estimate of production could be made from different published $P: B$ ratios, mean values were calculated and are reported here.

Standing crop biomass of Evadne nordmani was estimated assuming a mean individual weight of $2 \mu \mathrm{g} \mathrm{C}$ ( $\mathrm{P}$. Burkill pers. comm.). To estimate production a $P: B$ ratio for another cladoceran, Penilia avirostris (Greze 1978), was applied. For the appendicularian Oikopleura dioica linear measurements were converted to biomass using the equation given by Paffenhöfer

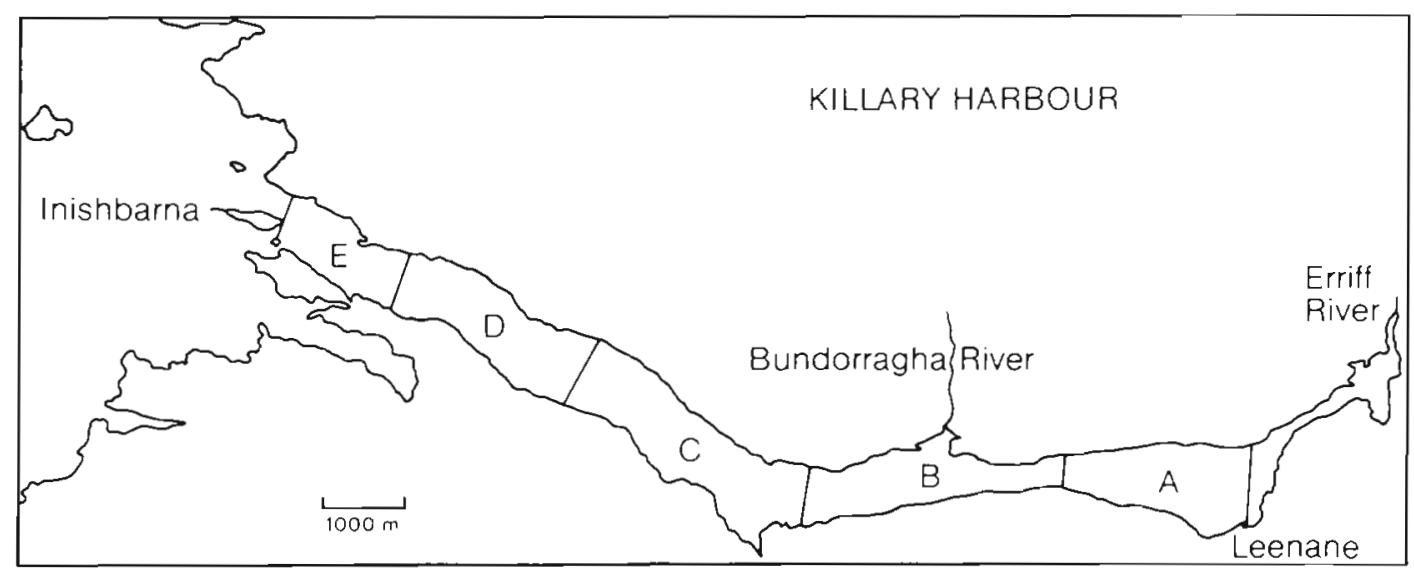

Fig. 1. Killary Harbour, west coast of Ireland, showing sectors 
(1976) and converted to carbon assuming a carbon content of 0.4 (Curl 1972). Production of $O$. dioica was estimated from the P:B ratio given by Zaika (1972).

For gelationous zooplankton the biomass of the ctenophore Pleurobrachia pileus was calculated from equations given by Hirota (1974). This incurred an error as the specimens were preserved in formalin and would have shrunk by up to $22 \%$ (Yip 1982). Carbon content was assumed to be 0.2 (Curl 1962) and production was calculated from the P:B ratio given for another ctenophore, Mnemiopsis sp. (Reeve \& Baker 1975). Daily production of medusae was approximated assuming that equal wet volumes of $P$. pileus and medusae have equal production rates.

Production of meroplanktonic bivalve and polychaete larvae was estimated on the assumption that together they accounted for the same fraction (0.13) of holozooplankton herbivore production, as in an Arctic fjord (McLaren 1969). Production of meroplanktonic cirrepede larvae was estimated by assuming a mean individual weight of $2.5 \mu \mathrm{g} \mathrm{C}$ for nauplii, $11 \mu \mathrm{g} \mathrm{C}$ for cyprids (Burkill pers. comm.) and a daily P:B ratio of 0.1 .

Benthos. Samples were taken between 7 August and 9 September 1980 with a diver-operated suction sampler (Barnett \& Hardy 1967). The sample was delivered into a bag with a $1 \mathrm{~mm}$ mesh suspended over the side of the support vessel. Details of samples taken in each sector are given in Table 2. Samples were returned to

Table 2. Details of macrobenthos samples taken in each sector

\begin{tabular}{|ccccc|}
\hline Sector & $\begin{array}{c}\text { Date } \\
(1980)\end{array}$ & $\begin{array}{c}\text { Quadrat area } \\
\left(\mathrm{m}^{2}\right)\end{array}$ & $\mathrm{n}$ & $\begin{array}{c}\text { Depth below } \\
\text { chart datum } \\
(\mathrm{m})\end{array}$ \\
\hline A & $19 \mathrm{Aug}$ & 0.25 & 5 & 1.4 \\
B & $18 \mathrm{Sep}$ & 0.25 & 5 & 4.7 \\
C & $11 \mathrm{Sep}$ & 0.25 & 5 & 6.5 \\
D & $12 \mathrm{Aug}$ & 0.25 & 5 & 12.2 \\
E & 7 Aug & 0.25 & 4 & 12.5 \\
\hline
\end{tabular}

the laboratory, sorted on a $1 \mathrm{~mm}$ mesh and preserved in $5 \%$ buffered formalin. They were later split according to taxonomic group and identified to species level.

Annelids, which tended to break up during sorting, and phoronids from each sample were pooled according to species and dried in tared aluminium foil dishes at $90^{\circ} \mathrm{C}$ for $24 \mathrm{~h}$, weighed, combusted in a muffle furnace at $500^{\circ} \mathrm{C}$ and re-weighed after cooling in a desiccator. Biomass, as ash-free dry weight (AFDW) of taxonomic groups from which large numbers of individuals were collected, was determined from regression equations relating AFDW ( $g$ ) to some linear dimension (LD) in $\mathrm{mm}$, which were derived from subsamples. LD was usually length, or disc diameter in the case of ophiuroids. Where small numbers of a species were present, or the size range was small, biomass of each individual was determined. AFDW determinations for molluscs included the organic component of the shell.

Mussels. Measurement of the production rates and carbon flow in wild and cultivated mussel populations in Killary Harbour was described by Rodhouse et al. (1985).

\section{RESULTS}

\section{Primary production and allochthonous carbon sources}

Flux of organic carbon in Killary Harbour, given by McMahon \& Patching (1984), is summarized in Table 3. They calculated a net export of 459 tonnes $C \mathrm{yr}^{-1}$ from Killary Harbour into the open sea. This is based on the calculated exchange of water at the mouth and the measured concentration of POC at 0, 5, 10 and $20 \mathrm{~m}$ in Sector E. Roden et al. (1987) show that a subsurface particulate maximum was frequently recorded at 5 or $10 \mathrm{~m}$ throughout the inlet in $1980 / 1981$. Booth (cited in Keegan \& Mercer 1986) also recorded a sonic scattering layer in May 1974 at approximately $5 \mathrm{~m}$. This particulate maximum coincided with the depth of the halocline. Calculations of carbon export will be greatly affected by the assumed depth of the upper, outwardflowing layer of water. McMahon \& Patching (1984) take this layer to include both the 0 and $5 \mathrm{~m}$ sampling points, thus often including the subsurface particulate maximum in the outward-flowing layer. The upper, outward-flowing layer is separated from the deeper inward-moving layer by a halocline (Booth op. cit.). An examination of the available salinity data (Hensey 1983) shows that on most sampling dates this halocline was $<5 \mathrm{~m}$ deep at Sector E. We therefore think that McMahon \& Patching overestimated carbon export from the system. It should be noted that these authors calculated that $75 \%$ of carbon export occurs between October and March, the period when phytoplankton growth is minimal.

Table 3. Annual flux of carbon in Killary Harbour from McMahon \& Patching (1985)

\begin{tabular}{|lc|}
\hline Source/sink & Tonnes carbon $\mathrm{yr}^{-1}$ \\
\hline Phytoplankton production & +1306 \\
Plankton respiration & +1531 \\
River-borne POC & +538 \\
River-borne active' DOC & +610 \\
Sedimentation & -463 \\
Flushing & -459 \\
\hline
\end{tabular}


Roden et al. (1987) suggest that phytoplankton is carried into Killary Harbour during the growing season, and Booth (cited in Keegan \& Mercer 1986) points out that particles in the lower inward-flowing layer would be retained. The large subsurface chlorophyll maxima revealed by Roden et al. (1987) may be caused by this mechanism. Therefore, there is little reason to believe that there is a net export of phytoplankton from the system; indeed there is some evidence to indicate that it is imported from coastal water.

Detritus derived from land runoff is probably exported. McMahon \& Patching (1984) show that large inputs of POC in freshwater runoff are associated with heavy rainfall; these conditions also favour a welldeveloped seaward-moving brackish layer (Roden et al. 1987).

Total annual macrophyte production is estimated to be 92 tonnes $C \mathrm{yr}^{-1}$ which is less than $10 \%$ of phytoplankton production. Applying the data of Johnstone et al. (1979) to this figure would result in approximately 50 tonnes $C \mathrm{yr}^{-1}$ being made available to other trophic levels. Total annual benthic microalgae production is estimated to be 51 tonnes $\mathrm{C} \mathrm{yr}^{-1}$.

\section{Zooplankton}

Biomass of holozooplankton and gelatinous zooplankton on each sampling date are given in Table 4. Estimated monthly production and total annual pro- duction for each species or group is given in Table 5. Total annual production of copepods, Evadne nordmanni, and Oikopleura dioica is estimated to 60.3 tonnes $\mathrm{C} \mathrm{yr}^{-1}$. Of this, $76.5 \%$ was contributed by copepods, of which calanoid copepods dominated with $64.3 \%$ of total holozooplankton production. The most prominent species responsible for calanoid copepod production were Pseudocalanus elongatus, Centropages hamatus, Temora longicornis, Acartia clausii, and to a lesser extent Paracalanus parvus and Isias clavipes. Cyclopoid copepods, although often very abundant, contributed only $12.3 \%$ of total holozooplankton production. Evadne nordmanni and Oikopleura dioica together contributed $23.5 \%$ of total holozooplankton production.

There were 2 peaks of gelatinous zooplankton production. The spring peak was dominated by medusae, mostly Phialella quadrata, but Pleurobrachia pileus was also relatively important. The autumn peak, which was lower than the spring peak was dominated by $P$. pileus. Of the estimated total annual production, $16.3 \%$ was contributed by medusae, mainly Phialella quadrata, and $37.5 \%$ was contributed by $P$. pileus. Estimated gelatinous zooplankton production was $27.0 \%$ of holozooplankton production.

Production by meroplankton was estimated to be 11.8 tonnes $\mathrm{C} \mathrm{yr}^{-1}$ so that the combined annual herbivorous holozooplankton and meroplankton produc-

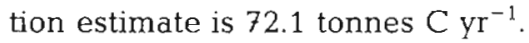

Table 4. Biomass of holozooplankton (copepods, Evadne nordmanni and Oikopleura dioica) and gelatinous zooplankton (medusae and Pleurobrachia pileus) in Killary Harbour

\begin{tabular}{|c|c|c|c|c|c|c|}
\hline \multirow[t]{2}{*}{ Date $(1981 / 82)$} & \multicolumn{3}{|c|}{ Holozooplankton } & \multicolumn{3}{|c|}{ Gelatinous zooplankton } \\
\hline & Killary tonnes $\mathrm{C}$ & $\mathrm{mg} \mathrm{C} \mathrm{m}{ }^{-3}$ & $\mathrm{mgC} \mathrm{m} \mathrm{m}^{-2}$ & Killary tonnes C & $\mathrm{mgCm} \mathrm{m}^{-3}$ & $\mathrm{mg} \mathrm{C} \mathrm{m} \mathrm{m}^{-2}$ \\
\hline $11 \mathrm{Feb}$ & 0.11 & 1.06 & 15.40 & 0.0001 & 0.001 & 0.02 \\
\hline 24 Feb & 0.22 & 2.12 & 20.80 & 0.0002 & 0.002 & 0.03 \\
\hline $10 \mathrm{Mar}$ & 0.20 & 1.93 & 28.40 & 0.0005 & 0.005 & 0.07 \\
\hline $26 \mathrm{Mar}$ & 0.36 & 3.48 & 60.56 & 0.0001 & 0.001 & 0.01 \\
\hline 9 Apr & 1.30 & 12.58 & 182.79 & 0.02 & 0.23 & 3.28 \\
\hline $23 \mathrm{Apr}$ & 2.65 & 25.64 & 372.55 & 0.63 & 6.10 & 36.32 \\
\hline 6 May & 7.62 & 70.25 & 1020.73 & 2.43 & 23.55 & 333.37 \\
\hline 21 May & 2.89 & 27.96 & 406.25 & 1.12 & 10.89 & 154.11 \\
\hline 4 Jun & 3.96 & 38.32 & 556.79 & 2.45 & 23.67 & 335.10 \\
\hline 17 Jun & 0.73 & 7.06 & 102.58 & 1.22 & 11.84 & 167.64 \\
\hline $8 \mathrm{Jul}$ & 5.07 & 49.06 & 712.84 & 0.07 & 0.67 & 9.45 \\
\hline $22 \mathrm{Jul}$ & 0.95 & 9.19 & 133.53 & 0.05 & 0.45 & 0.63 \\
\hline 5 Aug & 0.52 & 5.03 & 73.08 & 0.004 & 0.04 & 0.52 \\
\hline 19 Aug & 1.09 & 10.54 & 163.15 & 0.03 & 0.31 & 4.42 \\
\hline 23 Sep & 1.19 & 11.51 & 157.24 & 1.07 & 10.41 & 147.29 \\
\hline 7 Oct & 0.31 & 3.00 & 43.59 & 0.12 & 1.21 & 11.11 \\
\hline $22 \mathrm{Oct}$ & 0.82 & 7.93 & 115.22 & 0.51 & 4.94 & 63.94 \\
\hline 5 Nov & 1.02 & 9.87 & 143.41 & 0.01 & 0.07 & 0.99 \\
\hline $19 \& 24$ Nov & 0.57 & 5.51 & 80.06 & 0.10 & 0.95 & 13.46 \\
\hline $2 \mathrm{Dec}$ & 0.43 & 4.16 & 60.44 & 0.10 & 0.98 & 13.94 \\
\hline $6 \mathrm{Jan}$ & 0.28 & 2.71 & 39.38 & 0.001 & 0.01 & 0.13 \\
\hline
\end{tabular}


Table 5. Monthly production of zooplankton (tonnes C) in Killary Harbour

\begin{tabular}{|lcccccccccc|}
\hline Month & $\begin{array}{c}\text { Acartia } \\
\text { clausii }\end{array}$ & $\begin{array}{c}\text { Oithona } \\
\text { similis }\end{array}$ & $\begin{array}{c}\text { Oithona } \\
\text { nana }\end{array}$ & $\begin{array}{c}\text { Total } \\
\text { copepods } \\
\text { (mean) }\end{array}$ & $\begin{array}{c}\text { Evadne } \\
\text { nord- } \\
\text { manni }\end{array}$ & $\begin{array}{c}\text { Oikopleura } \\
\text { dioica }\end{array}$ & $\begin{array}{c}\text { Total } \\
\text { holozoo- } \\
\text { plankton }\end{array}$ & $\begin{array}{c}\text { Medusae } \\
\text { Pleuro- } \\
\text { brachia } \\
\text { pileus }\end{array}$ & $\begin{array}{c}\text { Total } \\
\text { gelatinous } \\
\text { zooplankton }\end{array}$ \\
\hline Feb & 0.007 & 0.0029 & 0.126 & 0.340 & 0.001 & 0.013 & 0.355 & 0.0004 & 0.0001 & 0.0005 \\
Mar & 0.083 & 0.124 & 0.054 & 0.688 & 0.018 & 0.070 & 0.776 & 0.0007 & 0.0004 & 0.001 \\
Apr & 0.344 & 0.387 & 0.215 & 4.862 & 1.568 & 1.278 & 7.708 & 1.006 & 0.007 & 1.013 \\
May & 1.729 & 0.378 & 0.077 & 15.391 & 2.247 & 0.012 & 17.65 & 5.116 & 0.736 & 5.853 \\
Jun & 1.660 & 0.063 & 0.048 & 5.429 & 0.205 & 0.613 & 6.247 & 4.021 & 1.497 & 5.518 \\
Jul & 2.826 & 0.694 & 0.791 & 7.824 & 3.575 & 1.362 & 12.761 & 0.005 & 0.192 & 0.197 \\
Aug & 0.235 & 0.057 & 1.297 & 2.451 & 1.186 & 0.174 & 3.811 & 0.001 & 0.071 & 0.072 \\
Sep & 2.671 & 0.104 & 1.109 & 4.299 & 0.037 & 1.189 & 5.525 & 0.006 & 1.956 & 1.962 \\
Oct & 0.051 & 0.029 & 1.070 & 1.372 & & 0.088 & 1.46 & 0.003 & 1.101 & 1.105 \\
Nov & 0.433 & 0.053 & 0.616 & 1.956 & & 0.412 & 2.368 & 0.002 & 0.202 & 0.204 \\
Dec & 0.202 & 0.034 & 0.409 & 1.210 & & 0.059 & 1.269 & 0.0006 & 0.346 & 0.347 \\
Jan & 0.039 & 0.061 & 0.208 & 0.532 & & 0.001 & 0.533 & 0.0005 & 0.003 & 0.003 \\
Total & 10.28 & 2.01 & 6.02 & 46.35 & 8.84 & 5.27 & 60.46 & 10.16 & 6.11 & 16.27 \\
\hline
\end{tabular}

\section{Benthos}

Regression equations relating biomass to LD in those species for which the regressions were calculated are given in Table 6 . In all cases there was significant correlation $(p<0.05)$ between biomass and LD.

Calculated values for biomass ( $\pm \mathrm{SE}$ ) for each species at each of the 5 Killary Harbour sectors are given in Table 7. Each species is designated a trophic role: deposit feeder, suspension feeder, deposit/suspension feeder, or predator according to its mode of feeding. The biomass, per $\mathrm{m}^{2}$ and per sector, of each phylum, according to its trophic role, is given in Table 8. Values for biomass in terms of carbon were estimated assuming that carbon content is 0.5 . This is the approximate carbon content for the ash-free dry soft tissue of Mytilus edulis (Rodhouse 1984a, Rodhouse et al. 1984b). The biomass of single large specimens was not included in the calculations for Table 8 because we consider that this would lead to an overestimate of total benthic biomass. The total standing crop biomass of

Table 6. Values for intercepts (A) and slopes (b) in regression equations relating biomass ( $\mathrm{g}$ AFDW) of macrobenthos species to linear dimension (LD $\mathrm{mm}$ ): $\log _{10} \mathrm{AFDW}=\mathrm{A}+$ blog $\log _{10} \mathrm{LD}$

\begin{tabular}{|llcccc|}
\hline Phylum & Species & A & b & r & df \\
\hline Mollusca & Abra nitida & -5.54 & 3.23 & 0.942 & 14 \\
& Myrtea spinifera & -6.16 & 3.83 & 0.972 & 12 \\
& Turitella communis & -7.02 & 3.84 & 0.948 & 18 \\
Echino- & Amphiura chiaje & -4.15 & 2.76 & 0.920 & 20 \\
dermata & & & & & \\
Sipuncu- & Golfingia elongata & -4.96 & 2.07 & 0.933 & 12 \\
lida & Golfingia procera & -6.83 & 2.96 & 0.940 & 11 \\
\hline
\end{tabular}

macrobenthic infauna in Killary Harbour is calculated to be 44.2 tonnes AFDW or approximately 22.1 tonnes of organic carbon. Standing crop biomass increases towards the mouth of the inlet and this is associated with a general increase in the numbers of species present (Table 9).

The community in Sector $A$ is impoverished and dominated by the mollusc Abra nitida whilst in Sectors B, C, D and E there is a richer boreal Amphiura chiaje community characteristic of deeper water elsewhere (Thorson 1957, Buchanan 1963). Estimates of annual production per unit area and for each sector, in terms of carbon, are given in Table 10. These estimates are made assuming an annual P:B ratio of 2.5 for Sector A and 0.4 for Sectors B, C, D and E. These ratios were taken from Sanders (1956) for a Nephtys/Yoldia community in Long Island Sound, USA, which is considered an iso-community of the Abra community of Thorson (1958), and from Buchanan \& Warwick (1974) for a Briopsis/Amphiura community off the east coast of Northumberland, UK. These ratios reflect the general observation that for Amphiura and Venus communities the P:B ratio is low and in Abra communities it is high (Warwick et al. 1978).

\section{Mussels}

Total annual production values and carbon flow for wild and cultivated mussels in Killary Harbour from Rodhouse et al. (1985) are given in Table 11. The data for cultured mussels are based on a total of $1239 \mathrm{~m}^{2}$ of production rafts, which was the extent of mussel culture in 1982 when data collection for this research was ended. The data for cultivated mussels is actually for a 14 mo period, September to November 1 yr later, but it includes only one production season. 


\section{Carbon budget}

A carbon flow diagram for the Killary Harbour system is shown in Fig. 2. Symbols are taken from Odum (1972). Particulate carbon input to the system is estimated to be: phytoplankton, 1306 tonnes $\mathrm{C} \mathrm{yr}^{-1}$; macroalgae, 50 tonnes $\mathrm{C} \mathrm{yr}^{-1}$; benthic microalgae, 51 tonnes $\mathrm{C} \mathrm{yr}^{-1}$; river carbon, 500 tonnes $\mathrm{C} \mathrm{yr}^{-1}$; making a total of 1907 tonnes $\mathrm{C} \mathrm{Yr}^{-1}$.

This carbon is consumed by zooplankton, wild mussels, cultured mussels, benthos, bacteria and mi- crozooplankton. Production of herbivorous zooplankton is 72 tonnes $\mathrm{C} \mathrm{yr}^{-1}$; assuming a $20 \%$ transfer efficiency (Parsons et al. 1977, Walsh 1981, Walsh et al. 1981) 380 tonnes $\mathrm{C} \mathrm{yr}^{-1}$ are required for zooplankton food. In 1981 there were $1239 \mathrm{~m}^{2}$ of mussel rafts and, assuming a consumption of 0.06 tonnes $\mathrm{C} \mathrm{m}^{-2} \mathrm{yr}^{-1}, 68$ tonnes $\mathrm{C} \mathrm{yr}^{-1}$ would be consumed. Wild mussels are estimated to consume less than 10 tonnes $\mathrm{C} \mathrm{yr}^{-1}$. Phytoplankton respiration consumes a proportion of the available carbon, 10 to $40 \%$ of total carbon production (Burris 1980). In making the present calculations a

Table 7. Biomass (AFDW: $\mathrm{mg} \mathrm{m}^{-2} \pm \mathrm{SE}$ ) of each benthic species in each sector

\begin{tabular}{|c|c|c|c|c|c|c|}
\hline \multirow[t]{2}{*}{ Species } & \multirow[t]{2}{*}{ Trophic role } & \multicolumn{5}{|c|}{ Sector } \\
\hline & & A & $\mathrm{B}$ & C & $\mathrm{D}$ & $E$ \\
\hline \multicolumn{7}{|l|}{ Mollusca } \\
\hline Nucula turgida & Deposit & & 3.2 & $12.6 \pm 4.0$ & $37.3 \pm 18.4$ & 18.5 \\
\hline Abra nitida & Deposit & $199.2 \pm 30.2$ & & & & \\
\hline Thyasira flexuosa & $\begin{array}{l}\text { Deposit/ } \\
\text { Suspension }\end{array}$ & & & & & $53.3 \pm 39.7$ \\
\hline Myrtea spinifera & $\begin{array}{l}\text { Deposit/ } \\
\text { Suspension }\end{array}$ & & $21.3 \pm 14.7$ & $38.4 \pm 15.2$ & $36.0 \pm 15.1$ & $112.3 \pm 51.5$ \\
\hline Dosinia exoleta & Suspension & & & $162.6 \pm 69.9$ & $46.9 \pm 34.9$ & $39.8 \pm 27.2$ \\
\hline Venus ovata & Suspension & & & 3.6 & & 8.0 \\
\hline Mya truncata & Suspension & & & & & 185.2 \\
\hline Ensis siliqua ${ }^{*}$ & Suspension & & & & & 5706.4 \\
\hline Turritella communis & Suspension & & $28.2 \pm 25.3$ & $387.0 \pm 168.9$ & $5799.6 \pm 2969.3$ & $2670.8 \pm 672.2$ \\
\hline \multicolumn{7}{|l|}{ Annelida } \\
\hline Melinna palmata & Deposit & $17.3 \pm 1.8$ & $173.2 \pm 66.5$ & $674.4 \pm 99.3$ & $11.3 \pm 8.4$ & $497.2 \pm 73.4$ \\
\hline Amphicteus gunneri & Deposit & & 27.0 & $19.6 \pm 8.4$ & $131.5 \pm 43.2$ & \\
\hline Notomastus latericeus & Deposit & 51.2 & $573.2 \pm 163.3$ & $130.9 \pm 74.6$ & 70.3 & $188.8 \pm 64.4$ \\
\hline Owenia fusiformis & Suspension & & & $23.7 \pm 14.5$ & 5.2 & $946.2 \pm 640.8$ \\
\hline Nephthys hystricus & Predator & $105.6 \pm 56.5$ & $770.8 \pm 166.4$ & $176.8 \pm 51.7$ & $188.0 \pm 92.8$ & $448.0 \pm 123.8$ \\
\hline Nephthys hombergi & Predator & $149.6 \pm 20.6$ & & & & \\
\hline Marphysa belli & Predator & & 330.0 & & & \\
\hline Hyalinoecia bilineata & Predator & & & $1175.2 \pm 608.2$ & & \\
\hline Stenelais boa & Predator & & & 91.6 & $58.4 \pm 39.7$ & $269.2 \pm 66.2$ \\
\hline \multicolumn{7}{|l|}{ Phoronida } \\
\hline Phoronis muelleri & Suspension & $3.5 \pm 2.2$ & $9.8 \pm 5.8$ & $378.0 \pm 100.2$ & $7.8 \pm 6.3$ & $497.2 \pm 36.8$ \\
\hline \multicolumn{7}{|l|}{ Echinodermata } \\
\hline Amphiura chiajei & Deposit & & $148.0 \pm 98.1$ & 202.4 & $2428.8 \pm 343.3$ & $2154.8 \pm 282.2$ \\
\hline Cuccumaria elongata & Deposit & & & & & $568.3 \pm 331.2$ \\
\hline Thyone fusus " & Deposit & & & $4388.5 \pm 2912.8$ & & \\
\hline \multicolumn{7}{|l|}{ Crustacea } \\
\hline Upogebia deltaura & Suspension & & & & & 1167.6 \\
\hline Hyppolyte sp. & Deposit & & & $78.9 \pm 32.5$ & & \\
\hline \multicolumn{7}{|l|}{ Cnidaria } \\
\hline Edwardsia callimorpha & Predator & & & $2083.6 \pm 441.8$ & & $511.8 \pm 208.5$ \\
\hline \multicolumn{7}{|l|}{ Sïpunculida } \\
\hline Golfingia elongata & Deposit & & & $932.4 \pm 310.2$ & 13.6 & $256.5 \pm 219.5$ \\
\hline Golfingia procera & Deposit & & & $204.8 \pm 103.2$ & 4.0 & 46.0 \\
\hline Golfingia vulgaris & Deposit & & & $511.0 \pm 421.7$ & 18.0 & 3641.2 \\
\hline Phascolion strombi & Deposit & & & & & $3.6 \pm 1.4$ \\
\hline \multicolumn{7}{|l|}{ Rhyncocoela } \\
\hline Nemertinea sp & Predator & & & & & $39.0 \pm 22.6$ \\
\hline
\end{tabular}


Table 8. Biomass (AFDW and estimated carbon) of major benthic taxa in each sector (biomass of single, or 2 only, large specimens shown in Table 7 omitted in calculating biomass in this table

\begin{tabular}{|c|c|c|c|c|c|c|}
\hline Sector & Taxon & Trophic role & $\operatorname{AFDW}\left(\mathrm{mg} \mathrm{m}^{-2}\right)$ & $\begin{array}{l}\text { AFDW (g) } \\
\text { for sector }\end{array}$ & Carbon $\left(\mathrm{mg} \mathrm{m}^{-2}\right)$ & $\begin{array}{l}\text { Carbon }(\mathrm{g}) \\
\text { for sector }\end{array}$ \\
\hline A & $\begin{array}{l}\text { Mollusca } \\
\text { Annelida } \\
\text { Phoronida }\end{array}$ & $\begin{array}{l}\text { Deposit } \\
\text { Deposit } \\
\text { Predator } \\
\text { Suspension }\end{array}$ & $\begin{array}{r}199.2 \\
68.5 \\
255.2 \\
3.5\end{array}$ & $\begin{array}{l}2.7 \times 10^{5} \\
9.2 \times 10^{4} \\
3.4 \times 10^{5} \\
4.7 \times 10^{3}\end{array}$ & $\begin{array}{r}99.6 \\
34.3 \\
127.6 \\
1.8\end{array}$ & $\begin{array}{l}1.4 \times 10^{5} \\
4.6 \times 10^{4} \\
1.7 \times 10^{5} \\
2.4 \times 10^{3}\end{array}$ \\
\hline$B$ & $\begin{array}{l}\text { Mollusca } \\
\text { Annelida } \\
\text { Phoronida } \\
\text { Echinodermata }\end{array}$ & $\begin{array}{l}\text { Deposit } \\
\text { Dep/sus } \\
\text { Suspension } \\
\text { Deposit } \\
\text { Predator } \\
\text { Suspension } \\
\text { Deposit }\end{array}$ & $\begin{array}{r}3.2 \\
21.3 \\
28.2 \\
773.2 \\
1100.8 \\
9.8 \\
148.0\end{array}$ & $\begin{array}{r}4.5 \times 10^{3} \\
3.0 \times 10^{4} \\
4.0 \times 10^{4} \\
1.09 \times 10^{6} \\
1.5 \times 10^{6} \\
1.4 \times 10^{4} \\
2.1 \times 10^{5}\end{array}$ & $\begin{array}{r}1.6 \\
10.7 \\
14.1 \\
386.6 \\
550.4 \\
4.9 \\
74.0\end{array}$ & $\begin{array}{l}2.3 \times 10^{3} \\
1.5 \times 10^{4} \\
2.0 \times 10^{4} \\
5.5 \times 10^{5} \\
8.0 \times 10^{5} \\
7.0 \times 10^{3} \\
1.1 \times 10^{5}\end{array}$ \\
\hline C & $\begin{array}{l}\text { Mollusca } \\
\text { Annelida } \\
\text { Phoronida } \\
\text { Echinodermata } \\
\text { Crustacea } \\
\text { Cnidaria } \\
\text { Sipunculida }\end{array}$ & $\begin{array}{l}\text { Deposit } \\
\text { Dep/sus } \\
\text { Suspension } \\
\text { Deposit } \\
\text { Suspension } \\
\text { Predator } \\
\text { Suspension } \\
\text { Deposit } \\
\text { Deposit } \\
\text { Predator } \\
\text { Deposit }\end{array}$ & $\begin{array}{r}12.6 \\
38.4 \\
553.2 \\
824.9 \\
23.7 \\
1443.6 \\
378.0 \\
202.4 \\
78.9 \\
2083.6 \\
1648.2\end{array}$ & $\begin{array}{l}2.7 \times 10^{4} \\
8.3 \times 10^{4} \\
1.2 \times 10^{6} \\
1.8 \times 10^{6} \\
5.1 \times 10^{4} \\
3.1 \times 10^{6} \\
8.2 \times 10^{5} \\
4.4 \times 10^{5} \\
1.7 \times 10^{5} \\
4.5 \times 10^{6} \\
3.6 \times 10^{6}\end{array}$ & $\begin{array}{r}6.3 \\
19.2 \\
276.6 \\
412.5 \\
11.9 \\
721.4 \\
169.0 \\
101.2 \\
39.5 \\
1041.8 \\
824.1\end{array}$ & $\begin{array}{l}1.4 \times 10^{4} \\
4.2 \times 10^{4} \\
6.0 \times 10^{5} \\
9.0 \times 10^{5} \\
2.6 \times 10^{4} \\
1.6 \times 10^{6} \\
4.1 \times 10^{5} \\
2.2 \times 10^{5} \\
9.0 \times 10^{4} \\
2.3 \times 10^{6} \\
1.8 \times 10^{6}\end{array}$ \\
\hline$D$ & $\begin{array}{l}\text { Mollusca } \\
\text { Annelida } \\
\text { Phoronida } \\
\text { Echinodermata } \\
\text { Sipunculida }\end{array}$ & $\begin{array}{l}\text { Deposit } \\
\text { Dep/sus } \\
\text { Suspension } \\
\text { Deposit } \\
\text { Suspension } \\
\text { Predator } \\
\text { Suspension } \\
\text { Deposit } \\
\text { Deposit }\end{array}$ & $\begin{array}{r}37.3 \\
36.0 \\
5846.5 \\
213.1 \\
5.2 \\
246.4 \\
7.8 \\
2428.8 \\
35.6\end{array}$ & $\begin{array}{l}5.3 \times 10^{4} \\
5.2 \times 10^{4} \\
8.4 \times 10^{6} \\
3.0 \times 10^{5} \\
7.4 \times 10^{4} \\
3.5 \times 10^{5} \\
1.1 \times 10^{4} \\
3.5 \times 10^{6} \\
5.1 \times 10^{4}\end{array}$ & $\begin{array}{r}18.7 \\
18.0 \\
2923.3 \\
106.6 \\
2.6 \\
123.2 \\
3.9 \\
1214.4 \\
17.8\end{array}$ & $\begin{array}{l}2.7 \times 10^{4} \\
2.6 \times 10^{4} \\
4.2 \times 10^{6} \\
1.5 \times 10^{5} \\
3.7 \times 10^{4} \\
1.8 \times 10^{5} \\
6.0 \times 10^{3} \\
1.8 \times 10^{6} \\
2.6 \times 10^{4}\end{array}$ \\
\hline $\mathrm{E}$ & $\begin{array}{l}\text { Phoronida } \\
\text { Echinodermata } \\
\text { Cnidaria } \\
\text { Sipunculida } \\
\text { Rhynchocela }\end{array}$ & $\begin{array}{l}\text { Deposit } \\
\text { Dep/sus } \\
\text { Suspension } \\
\text { Deposit } \\
\text { Suspension } \\
\text { Predator } \\
\text { Suspension } \\
\text { Deposit } \\
\text { Predator } \\
\text { Deposit } \\
\text { Predator }\end{array}$ & $\begin{array}{r}18.5 \\
165.6 \\
2718.6 \\
686.0 \\
946.2 \\
717.2 \\
497.2 \\
2723.1 \\
511.8 \\
3947.3 \\
39.0\end{array}$ & $\begin{array}{l}1.8 \times 10^{4} \\
1.6 \times 10^{5} \\
2.6 \times 10^{6} \\
6.6 \times 10^{5} \\
9.0 \times 10^{5} \\
6.8 \times 10^{5} \\
4.7 \times 10^{5} \\
2.6 \times 10^{6} \\
4.9 \times 10^{5} \\
3.8 \times 10^{6} \\
3.7 \times 10^{4}\end{array}$ & $\begin{array}{r}9.2 \\
82.8 \\
1359.3 \\
343.0 \\
473.1 \\
353.6 \\
248.6 \\
1361.6 \\
255.9 \\
1973.7 \\
19.5\end{array}$ & $\begin{array}{l}9.0 \times 10^{3} \\
8.0 \times 10^{4} \\
1.3 \times 10^{6} \\
3.3 \times 10^{5} \\
4.5 \times 10^{5} \\
3.4 \times 10^{5} \\
2.4 \times 10^{5} \\
1.3 \times 10^{6} \\
2.5 \times 10^{5} \\
1.9 \times 10^{6} \\
1.9 \times 10^{4}\end{array}$ \\
\hline
\end{tabular}

Table 9. Numbers of benthic species and biomass in each sector

\begin{tabular}{ccc|}
\hline Sector & No. of species & Biomass $\left(\mathrm{g} \mathrm{AFDW} \mathrm{m}^{-2}\right)$ \\
\hline A & 6 & 0.53 \\
B & 10 & 2.08 \\
C & 20 & 7.28 \\
D & 15 & 8.86 \\
E & 23 & 12.96 \\
\hline
\end{tabular}

value of $15 \%$ was used which reflects the reported values found in many phytoplankton populations. The remaining carbon produced by the phytoplankton is consumed either directly by the microzooplankton, sediments as detritus or is flushed out at the mouth of the harbour. We conclude that carbon production by the phytoplankton is partitioned thus: zooplankton, 380 tonnes $\mathrm{C} \mathrm{yr}^{-1}$; cultured mussels, 68 tonnes $\mathrm{C} \mathrm{yr}^{-1}$; wild mussels, 10 tonnes $\mathrm{C} \mathrm{yr}^{-1}$; phytoplankton respira- 
Table 10. Biomass and estimated production of macrobenthic infauna in Killary Harbour

\begin{tabular}{|cccccc}
\hline Sector & Biomass $\left(\mathrm{g} \mathrm{C} \mathrm{m}^{-2}\right)$ & $\begin{array}{c}\text { Biomass in sector } \\
\text { (tonnes C) }\end{array}$ & $\mathrm{P}: \mathrm{B}$ & $\begin{array}{c}\text { Estimated production } \\
\left(\mathrm{g} \mathrm{C} \mathrm{m}^{-2} \mathrm{yr}^{-1}\right)\end{array}$ & $\begin{array}{c}\text { Estimated production in } \\
\text { sector (tonnes C) }\end{array}$ \\
\hline A & 0.26 & 0.36 & 2.5 & 0.65 & 0.90 \\
B & 1.04 & 1.50 & 0.4 & 0.42 & 0.60 \\
D & 3.64 & 8.00 & 0.4 & 1.46 & 3.20 \\
E & 4.43 & 6.45 & 0.4 & 1.77 & 2.58 \\
Total & 6.48 & 6.22 & 0.4 & 2.59 & 2.48 \\
\hline
\end{tabular}

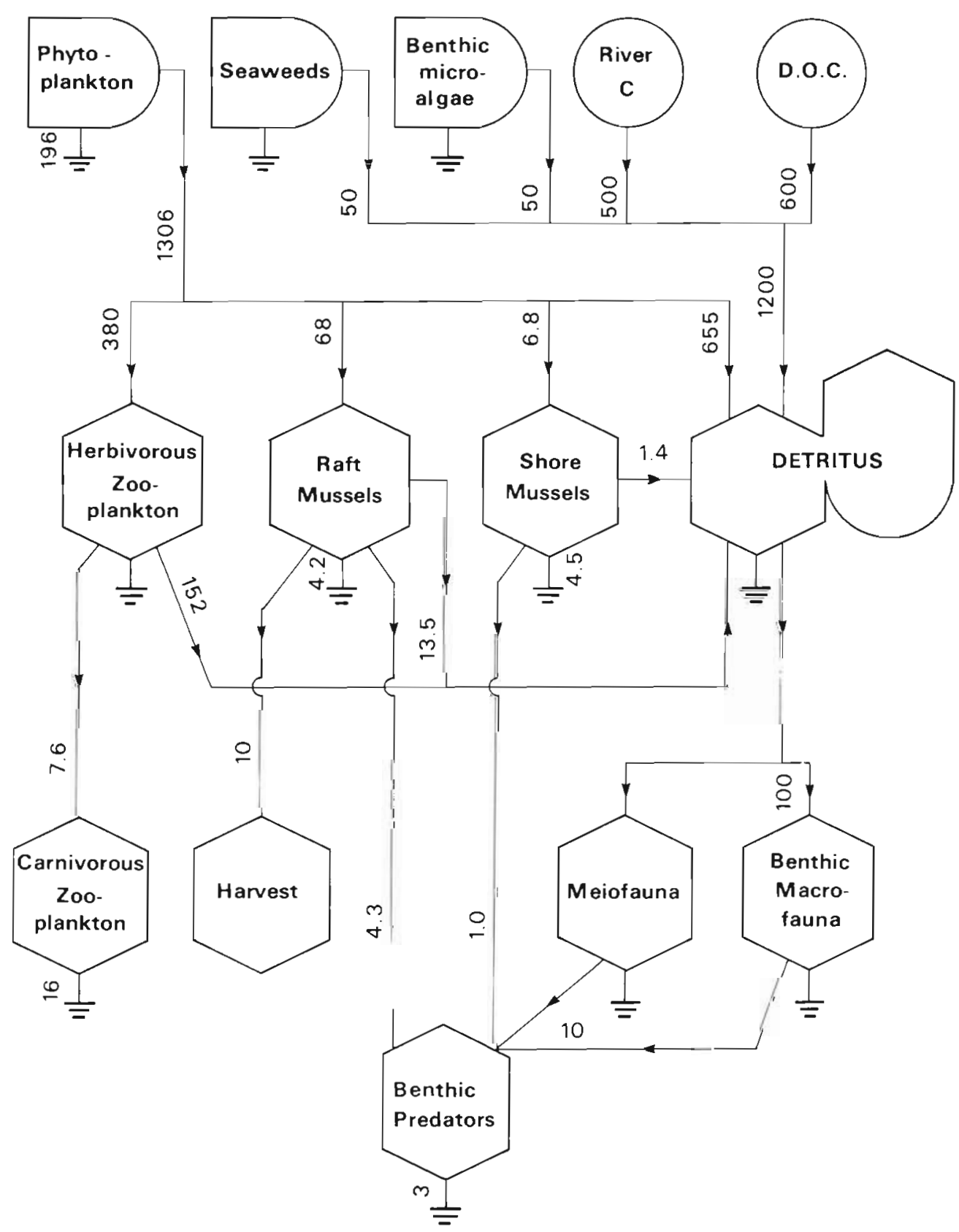

Fig. 2. Carbon flow diagram for Kullary Harbour. Numbers are tonnes ${\mathrm{C} \mathrm{yr}^{-1}}^{-1}$ 
Table 11. Total annual production and carbon flow (tonnes $\mathrm{C} \mathrm{yr}^{-1}$ ) for wild and cultured mussels in Killary Harbour

\begin{tabular}{|lccccccc|}
\hline & Consumption & $\begin{array}{c}\text { Production } \\
\text { (including shell) }\end{array}$ & Gametes & $\begin{array}{c}\text { Production }+ \\
\text { gametes }\end{array}$ & Respiration & Elimination & Faeces \\
\hline Wild & 6.9 & - & - & 1.0 & 4.5 & - & 1.4 \\
Cultured & 68.4 & 10.8 & 2.0 & 12.8 & 42.4 & 5.3 & 13.8 \\
\hline
\end{tabular}

tion, 196 tonnes $\mathrm{C} \mathrm{yr}^{-1}$; detritus, 652 tonnes $\mathrm{C} \mathrm{yr}^{-1}$; making a total of 1306 tonnes $\mathrm{C} \mathrm{yr}^{-1}$.

The large fraction which is not consumed but goes to the detritus pool reflects the finding of other workers who estimate that up to $60 \%$ of primary production is not directly consumed (Rosenberg et al. 1977, Walsh 1981).

The remaining carbon sources, river input, macroalgae and benthic microalgae, are not considered here to contribute significantly to mussel or zooplankton production but supply benthic fauna and microzooplankton. The detritus pool receives the following known inputs: river carbon, 500 tonnes $\mathrm{C} \mathrm{yI}^{-1}$; macroalgae, 50 tonnes $\mathrm{C} \mathrm{yr}{ }^{-1}$; zooplankton faeces, 152 tonnes $\mathrm{C} \mathrm{yr}{ }^{-1}$; mussel faeces, 14 tonnes $\mathrm{C} \mathrm{yr}^{-1}$; phytoplankton, 652 tonnes $\mathrm{C} \mathrm{yr}^{-1}$; making a total of 1368 tonnes $\mathrm{C} \mathrm{yr}{ }^{-1}$.

The fate of the detritus pool is complicated because it is derived from 2 very different major sources: phytoplankton which is easily metabolised, produced mainly in the growing season and probably not exported, and detrital carbon, carried into Killary Harbour by runoff. It is present throughout the year but is most prominent during periods of high rainfall. When the upper layer is well developed it is probably exported beyond the inlet. However, Keary (cited in Keegan \& Mercer 1986) has shown that the bottom sediments in the inner part of the inlet between Sectors $A$ and $C$ contain up to $9 \%$ organic carbon, mainly in the form of humified plant remains. This shows that sedimentation of allochthonous carbon occurs within the Harbour and that this material is not easily metabolised.

The detrital pool can therefore be divided into 2 parts: an easily metabolised fraction which is consumed by the zooplankton and benthos and a refractory part which is incorporated into the sediments or exported. McMahon \& Patching (1984) show that a total of 1531 tonnes $\mathrm{C} \mathrm{yr}^{-1}$ are metabolised; of this, phytoplankton respiration accounts for 196 tonnes $C$ $\mathrm{yr}^{-1}$. The remaining 1306 tonnes $\mathrm{C} \mathrm{yr}^{-1}$ are due to microzooplankton and bacterial respiration. Assuming no net export of carbon, 62 tonnes $\mathrm{C} \mathrm{yr}^{-1}$ are then available for sedimentation from the detrital pool.

McMahon \& Patching (1984) postulate a supply of metabolically available dissolved organic carbon from land runoff. In addition, the import of phytoplankton in summer may augment the available detrital carbon pool (Roden et al. 1987). Neither of these sources are easily measured but together with the measured carbon sources, the detrital pool is large enough to support the estimated consumption.

The benthic community was not studied as intensively as the plankton so estimates of carbon requirements are probably less accurate. However, the figure for macrobenthos production, 10 tonnes $\mathrm{C} \mathrm{yr}^{-1}$, is probably reasonable and would require an input of 100

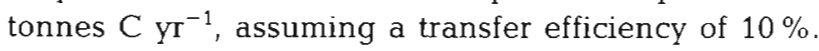
Meiobenthos have not been estimated.

\section{DISCUSSION}

In order to obtain the carbon budget presented here a number of estimations and approximations have been necessary. The use of $\mathrm{P}: \mathrm{B}$ ratios in calculating production by zooplankton and benthos is open to legitimate criticism because in doing this we infer that conditions at our study site are similar to those in different habitats, in different geographical areas. Also, with the time and manpower available we were not able to sample every sub-component of the system with the same intensity. Sampling was carried out to a degree considered appropriate to the importance of each sub-system, thus the benthos was only sampled at one time of year, late summer, when this community may well be at near maximum biomass. However in view of the relative importance of the benthos in the overall budget we do not consider that this would lead to serious error. In spite of such weaknesses we feel sufficiently confident in our data to attempt to predict the potential carrying capacity of the Killary Harbour system for intensive bivalve culture and to make some general predictions about the effects of this industry.

If total mussel yield ( $Y$ ) depends on the amount of available food and the conversion efficiency of the mussels then:

$$
Y=y \cdot F / f
$$

where $\mathrm{y}=$ yield per unit area (tonnes $\left.\mathrm{C} \mathrm{m}^{-2} \mathrm{yr}^{-1}\right)$; $\mathrm{f}=$ food consumed per unit area (tonnes $\mathrm{C} \mathrm{m}^{-2} \mathrm{yr}^{-1}$ ); and

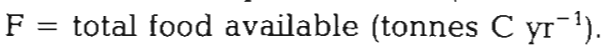

Rodhouse et al. (1985) have shown that for $1 \mathrm{~m}^{2}$ of 
mussel raft, 0.56 tonnes $\mathrm{C} \mathrm{yr}^{-1}$ are consumed and 0.008 tonnes $\mathrm{C} \mathrm{yr}^{-1}$ (mussels) can be harvested. We assume in the following calculations that the carbon content of live mussels is $2.95 \%$. Tenore \& Gonzalez (1976) and Hanson et al. (1986) have shown that cultured mussels in the Ria de Arosa, Northern Spain, compete with zooplankton for the available food supply. In Killary Harbour, if cultured mussels were to consume all the carbon now consumed by zooplankton as well as that now consumed by cultured mussels $(360+68$ tonnes $C$ $\mathrm{yr}^{-1}$ ) annual yield would be 2073 tonnes live weight.

Rodhouse et al. (1985) have also shown that during flood and ebb tide approximately $50 \%$ of particulates are cleared from suspension by mussel rafts. This was in close agreement with Cabañas et al. (1976) who give a value of $60 \%$ removal. If $F$ in Eqn (1) equals 650 tonnes ${\mathrm{C} \mathrm{yr}^{-1}}^{-1}$, half the total phytoplankton production, then annual yield would be 3162 tonnes live weight.

If the suggestion is correct that phytoplankton accumulate within the inlet from outside (Roden et al. 1987), then this process might supply additional food to the mussels cultured on ropes. However, it is difficult to quantify this process without a clearer understanding of the physical processes which govern it.

These calculations assume that the cultured mussels consume only phytoplankton. Widdows et al. (1979) suggested that detrital carbon and bacteria contribute to mussel nutrition and so it is possible that the above calculations underestimate potential yield. However, there is evidence that the hydrological structure of Killary Harbour is such that the major food resource available to mussels in suspended culture is phytoplankton (Rodhouse et al. 1984a, Roden et al. 1987) and so we conclude that the maximum annual yield of mussels is approximated by these calculations. Once annual yield exceeds about 3000 tonnes over half the primary productivity of the inlet will have been diverted to mussel culture. Beyond this, severe modifications of the environment and decreasing yields per unit area may be expected.

Large-scale mussel culture in coastal marine sites will inevitably cause environmental change. In calculating potential yield it has been assumed here that as yield increases, zooplankton will decrease. Such a substitution of the major grazing community can be expected to alter several aspects of the Killary Harbour system.

At present zooplankton grazing occurs throughout the system and associated processes, such as excretion of ammonia and deposition of faecal pellets, are widespread. By replacing dispersed zooplankton grazing by mussel grazing, concentrated in small areas, these processes will be concentrated resulting in areas of heavy sedimentation interspersed with areas of low sedimentation. This might be expected to alter the composition and distribution of the benthic fauna (Tenore et al. 1982, Rosenberg \& Loo 1983, Kaspar et al. 1985). Dahlback \& Gunnarsson (1981) report that sedimentation is increased 3-fold under mussel culture rafts compared to control sites in Sweden. The benthic community is dominated there by large white mats of the sulphate-reducing bacteria Beggiatod. This does not occur in Killary Harbour probably because of differences in the depth of water and tidal current flow (Rodhouse et al. 1985).

Here, potential mussel yield was calculated as carbon but Roden et al. (1987) have shown that primary production was sometimes limited in summer by nitrogen, consequently any effects of mussel culture on the nitrogen budget may be considerable. Because nitrogen is limiting, its removal as mussel tissue must be compensated by additional nitrogen if primary production is not to decline. The substitution of zooplankton grazing by mussel grazing may also alter the rates of nitrogen cycling and loss to the sediments.

Roden et al. (1987) have indicated that nitrate is continually supplied to Killary Harbour from both river runoff and from the sea. The estimated size of nitrate input is 278 tonnes $\mathrm{N} \mathrm{yr}^{-1}$ which exceeds the expected maximum quantity harvested, 26 tonnes $\mathrm{N} \mathrm{yr}^{-1}(=3200$ tonnes live weight) by an order of magnitude. Therefore nitrogen removal should not limit mussel production in Killary Harbour.

Mussel culture may also alter the rate of nitrogen cycling. If the rate were increased an increase in primary production might be expected and the possibility of local blooms of red tide organisms exists. While red tides have caused problems for mussel cultures elsewhere it is not established that these blooms are a result of cultivation. Paralytic and diarretic shellfish poisoning have been recorded from mussel growing areas including the Netherlands, Galicia (northwest Spain), and Bantry Bay (southwest Ireland) (Kat 1985, Fraga \& Sanchez 1985, Dunne pers. comm.). All these areas have a high natural phytoplankton production which is often a characteristic of red tide prone regions (Holligan 1985). This high productivity is also an advantage in mussel production. While mussel rafts reroute the flow of energy and materials they do not add extra nutrients to the ecosystem, unlike caged fish fed on prepared food. Any increase in algal production due to mussel cultivation will involve processes very different from eutrophication caused by an allochthonous nutrient supply.

Acknowledgements. We thank Professor Padraig $\mathrm{O}^{\circ}$ Ceidigh and Dr. John Mercer for providing research facilities at the Shellfish Research Laboratory and Duncan Brown, James O'Neill, and Michael Rowley for their assistance. Dr. Brendan Keegan loaned the airlift suction sampler and Drs. David McGrath and Brendan O'Connor assisted with benthic 
species identification. Mary Hensey and Thomas Ryan contributed considerably, in the field and in the laboratory, to the research described here and we thank them for their help. Many scientists criticised and discussed our research with us. We thank them all especially Drs. Brian Bayne, Rutger Rosenberg, Ken Tenore and John Walsh. The Killary Harbour Project was funded by the National Board for Science and Technology (Ireland) and Beirtreach Teoranta. Contribution No. 278 from The School of Marine Science, University College, Galway.

\section{LITERATURE CITED}

Burris, J. E. (1980). Respiration and photorespiration in marine algae. In: Falkowsky, P. G. (ed.) Primary productivity in the sea. Plenum Press, New York, p. 411-432

Barnett, P. O., Hardy, B. C. S. (1967). A diver-operated suction sampler for sand macrofaunas. Helgoländer wiss. Meeresunters. 15: 390-398

Buchanan, J. B. (1963). The bottom fauna communities and their sediment relationships off the coast of Northumberland. Oikos 14: 154-175

Buchanan, J. B., Warwick, R. M. (1974). An estimate of benthic macrofaunal production in the offshore mud of the Northumberland coast. J. mar. biol. A.ss. U. K. 54: 197-222

Cabañas, J. M., González, J. J., Marión, J., Pérez, A., Román, G. (1979). Estudio del mejillon y de su epifauna en los cultivos flotantes, de la Ría de Arosa. III. Observaciones previas sobre la retención de partículas y la biodeposición de una batea. Boln Inst. esp. Oceanogr. 5; 45-50

Curl, H. (1962). Analysis of carbon in marine plankton. J. mar Res. 20: 181-188

Dahlback, B., Gunnarsson, L. A. (1981). Sedimentation and sulfate reduction under a mussel culture. Mar. Biol. 63: 269-275

Durbin, E. G., Durbin, A. J. (1978). Length and weight relationships of Acartia clausii from Narragansett Bay, R. I. Limnol. Oceanogr. 23: 958-969

Evans, F. (1977). Seasonal density and production estimates of the commoner planktonic copepods of Northumberland coastal waters. Estuar. coast. mar. Sci. 5: 223-241

Fraga, S., Sanchez, F. J. (1985). Toxic and potentially toxic dinoflagellates found in Galician Rias (N. W. Spain). In: Anderson, D. M., White A. W., Baden, D. G. (ed.) Toxic dinoflagellates. Elsevier, Amsterdam, p. 51-54

Greze, V N. (1978). Production in animal populations. In: Kinne, O. (ed.) Marine ecology, Vol. IV, Dynamics. Wiley, Chichester, p. 89-114

Greze, V. N., Baldina, E. P., Bileva, O. K. (1968), Production of planktonic copepods in neritic zone of Black Sea. Okeanologiya 8: 1066-1070

Hanson, R. B., Alvarez-Ossorio, M. T., Cal, R., Campos, M. J., Roman, M., Santiago, G., Varela, M., Yoder, J. A. (1986). Plankton response following a spring upwelling event in the Ria de Arosa, Spain. Mar. Ecol. Prog. Ser. 32: 101-113

Hensey, M. P. (1983). Physical factors and oceanography of Killary Harbour. In: Establishing the potential yield of cultivated bivalve molluscs (mussels) in Killary Harbour: a model. Final report on HEIC project No. 11/79. Shellfish Research Laboratory, p. 9-39

Hirota, J. (1974). Quantitative natural history of Pleurobrachia bachei in Jolla Bight. Fish. Bull. U. S. 72: 295

Holligan, P. M. (1985). Marine dinoflagellate blooms growth strategies and environmental exploitation. In: Anderson, D. M., White A. W., Baden, D. G. (ed.) Toxic dinoflagellates. Elsevier, Amsterdam, p. 133-139

Johnstone, C. S., Jones, R. G., Hunt, R. D. (1979). A seasonal carbon budget for a laminarian population in a Scottish sea-loch. Helgoländer wiss. Meeresunters. 30: 527-545

Kaspar, Gillespie, P. A., Boyer, I. C., McKenzie, A. l. (1985). Effects of mussel aquaculture on the nitrogen cycle and benthic communities in Kenepuru Sound, Marlborough Sounds, New Zealand. Mar. Biol. 85: 127-136

Kat, M. (1985). Dinophysis acuminata blooms, the distinct cause of Dutch mussel poisoning. In: Anderson, D. M., White A. W., Baden, D. G. (ed.) Toxic dinoflagellates. Elsevier, Amsterdam, p. 73-77

Keegan, B. F., Mercer, J. P. (1986). An oceanographical survey of Killary Harbour on the the west coast of Ireland. Proc. R. Ir. Acad. $86 \mathrm{~B}: 1-70$

McLaren, I. A. (1969). Population and production ecology of zooplankton in Ogac lake, a landlocked fjord on Baffin Island. J. Fish. Res. Bd Can. 26: 1485-1559

McMahon, T. G., Patching, J. W. (1984). Fluxes of organic carbon in a fjord on the west coast of Ireland. Estuar. coast. Shelf Sci. 19: 205-215

Odum, H. T. (1972). An energy circuit language for ecological and social systems. In: Patten, B. C. (ed.) Systems analysis and simulation in ecology, Vol. 2. Academic Press, New York, p. 139-211

Omori, M. (1969). Weight and chemical composition of some important oceanic zooplankton in the North Pacific Ocean. Mar. Biol. 3: 4-10

Paffenhöfer, G. A. (1976). On the biology of Appendicularia of the southeastern North Sea. In: Persoone, G., Jaspers, E. (ed.) 10th European Symposium on Marine Biology, Ostend, Belgium, Vol. 2. Population dynamics. Universa Press, Wetteren, p. 437-455

Parsons, T. R., Takahashi, M., Hargrave, B. (1977). Biological oceanographic processes, 2nd edn. Pergamon Press, New York

Raine, R. C. T., Patching, J. W. P. (1980). Aspects of carbon and nitrogen cycling in a shallow marine environment. $J$. exp. mar Biol. Ecol. 47: 127-139

Raymont, J. E. G. (1980). Plankton and productivity in the oceans. Vol. I, Phytoplankton. Pergamon Press, Oxford

Reeve, M. R., Baker, L. D. (1975). Production of two planktonic carnivores (chaetognath and ctenophore) in south Florida inshore waters. Fish. Bull. U. S. 73: 238-248

Robertson, A. I. (1968). The continuous plankton recorder: a method for studying the biomass of calanoid copepods. Hull Bull. mar. Ecol. 6: 238-248

Roden, C. M., Rodhouse, P. G., Hensey, M. P., McMahon, T., Ryan, T. H., Mercer, J. P. (1987). Hydrography and the distribution of phytoplankton in Killary Harbour: a fjord in western Ireland. J. mar. biol. Ass. U. K. 67: in press

Rodhouse, P. G., Roden, C. M., Burnell, G. M., Hensey, M. P., McMahon, T., Ottway, B., Ryan, T. H. (1984a). Food resource, gametogenesis and growth of Mytilus edulis on the shore and in suspended culture: Killary Harbour, Ireland. J. mar. biol. A.ss. U. K. 64: 513-530

Rodhouse, P. G., Roden, C. M., Hensey, M. P., Ryan, T. H. (1984b). Resource allocation in Mytilus edulis on the shore and in suspended culture. Mar. Biol. 84: 27-34

Rodhouse, P. G., Roden, C. M., Hensey, M. P., Ryan, T. H. (1985). Production of mussels, Mytilus edulis, in suspended culture and estimates of carbon and nitrogen flow: Killary Harbour, Ireland. J. mar, biol. Ass. U. K. 65: 55-68

Rosenberg, R., Loo, L.-O. (1983). Energy-flow in a Mytilus edulis culture in western Sweden. Aquaculture 35: $151-161$

Rosenberg, R., Olsson, I., Olundh, E. (1977). Energy flow model of an oxygen-deficient estuary on the Swedish west coast. Mar. Biol. 42: 99-107 
Ryan, T. H., Rodhouse, P. G., Roden, C. M., Hensey, M. P. (1986). Zooplankton fauna of Killary Harbour: the seasonal cycle of abundance. J. mar. biol. Ass. U. K. 66 : $731-748$

Ryther, J. H. (1969). The potential of the estuary for shellfish production. Proc natn. Shellfish Ass. 59: 18-22

Sanders, H. L. (1956). The biology of marine bottom communities. Bul. Bingham oceanogr. Coll. 15: 344-414

Tenore, K. R., Boyer, L. G., Cal, R. M., Corral, J., GarciaFernandez, C., Gonzalez, N., Gonzalez-Gurriaran, E., Hanson, R. B., Iglesias, J., Krom, M., Lopez-Jamar, E., McLain, J., Pamatmat, M. M., Perez, A., Rhoads, D. C., de Santiago, G., Tietjen, J., Westrich, J., Windom, H. L. (1982). Coastal upwelling in the Rias Bajas, NW Spain: contrasting the benthic regimes of the Rias de Arosa and de Muros. J. mar. Res. 40: 701-772

Tenore, K. R., Gonzalez, N. (1976). Food chain patterns in the Ria de Arosa, Spain: an area of intense mussel aquaculture. In: Persoone, G., Jaspers, E. (ed.) 10th European Symposium on Marine Biology, Ostend, Belgium, Vol. 2. Population dynamics. Universa Press, Wetteren, p. 601-619
Thorson, G. (1957). Bottom communities (sublittoral or shallow shelf). Mem. geol. Soc. Am. 67: 461-534

Uye, S. (1982). Population dynamics and production of Acartia clausii Giesbrecht (Copepoda:Calanoida) in inlet waters. J. exp. mar. Biol. Ecol. 57: 55-83

Walsh, J. J. (1981). Shelf-sea ecosystems. In: Longhurst, A. R. (ed.) Analysis of marine ecosystems. Academic Press, New York, p. 159-196

Walsh, J. J., Rowe, G. T., Iverson, R. L., McRoy, C. P. (1981). Biological export of shelf carbon is a sink of the global $\mathrm{CO}_{2}$ cycle. Nature, Lond. 291: 196-201

Warwick, R. M., George, C. L., Davies, J. R. (1978). Annual macrofauna production in a Venus community. Estuar. coast. mar. Sci. 7: 215-241

Widdows, J., Fieth, P., Worrall, C. M. (1979). Relationships between seston, available food and feeding activity in the common mussel Mytilus edulis. Mar. Biol. 50: 195-207

Yip, S. Y. (1982). A note on the effect of preserving ctenophores in formaldehyde-seawater. Ir. Nat. J. 20: $416-419$

Zaika, V. E. (1972). Specific production of aquatic invertebrates. Wiley, New York

This article was presented by Dr. R. M. Warwick; it was accepted for printing on December 22, 1986 